\title{
DEDICATED TOOL FOR IRRADIATION AND ELECTRICAL MEASUREMENT OF LARGE SURFACE SAMPLES ON THE BEAMLINE OF A 2.5 MEV PELLETRON ELECTRON ACCELERATOR: APPLICATION TO SOLAR CELLS
}

\author{
Jérémie Lefèvre*, Patrice Le Houedec, Jérôme Losco, Olivier Cavani, and Bruno Boizot \\ Laboratoire des Solides Irradiés, CNRS-UMR 7642, CEA-DRF-IRAMIS, Ecole Polytechnique, Université Paris-Saclay, \\ 91128 Palaiseau Cedex, France
}

\begin{abstract}
We designed a tool allowing irradiation of large samples over a surface of A5 size dimension by means of a 2.5 MeV Pelletron electron accelerator. in situ electrical measurements (I-V, conductivity, etc.) can also be performed, in the dark or under illumination, to study radiation effects in materials. Irradiations and electrical measurements are achievable over a temperature range from $100 \mathrm{~K}$ to $300 \mathrm{~K}$.

The setup was initially developed to test real-size triple junction solar cells at low temperature within the framework of the ESA's JUICE mission. It will be made available to users at the beginning of 2017.

After a brief description of the SIRIUS irradiation facility hosted at Laboratoire des Solides Irradiés (LSI), this paper gives detailed information about the Large Surface Irradiated-Cell (LSIC) device. Preliminary results obtained during the ongoing qualification phase of the setup are also discussed.
\end{abstract}

Key words: SIRIUS; electron irradiation; triple jonction solar cell; JUICE mission.

\section{INTRODUCTION}

\subsection{Laboratoire des Solides Irradiés}

The LSI laboratory at Ecole Polytechnique (Palaiseau, France) is a mixed unit affiliated to CEA, CNRS and Ecole Polytechnique. It studies fundamental properties of the solid state and its diverse interactions with radiation (photons, electrons, ions).

The laboratory has a $2.5 \mathrm{MeV}$ electron accelerator with the unique feature of having a large variety of in situ characterization techniques. This installation, opened in 2013, is called SIRIUS for Système d'IRradiation pour l'Innovation et les Utilisations Scientifiques.

\footnotetext{
*Email: jeremie.lefevre@ polytechnique.edu
}

\subsection{SIRIUS facility}

The SIRIUS facility is equipped with a new generation Pelletron accelerator that delivers monoenergetic electrons beams between $150 \mathrm{keV}$ and $2.5 \mathrm{MeV}$ with a current beam ranging from $150 \mathrm{nA}$ to $200 \mu \mathrm{A}$. It is equipped with two beamlines, and a host of equipment for online experiments, including two cryostats to study the low temperature electrical transport properties of irradiated materials. Room Temperature (RT) experiments include visible and near-infrared PhotoLuminescence (PL), cathodoluminescence, and Raman spectroscopy. Low temperature in situ electron paramagnetic resonance and PL will be installed on the SIRIUS beamline in 2017. Such a large variety of online equipments makes the SIRIUS facility a unique tool in the world.

The accelerator is planned to work more than 200 days per year. It is available to a large community of scientists and industrials from France and abroad. It has also been developed to be complementary with other irradiation facilities like ion beams for example. SIRIUS is actually part of the french national accelerator network for the study of materials under irradiation (EMIR). [1]

The scientific topics currently studied around the accelerator are :

- Effects of electron irradiation on material properties (mechanical, optical, magnetic and electrical transport in high TC superconductors);

- Ageing under irradiation;

- Characterization of induced defects (type of defects, displacement thresholds);

- Radio-grafting of polymers (in particular applications to biomaterials);

- Corrosion under irradiation.

Electron irradiation can also be used to simulate the radiative environment in space. For this purpose, the LSIC device has been developed to study the degradation of 
electrical properties in space solar cells. This setup will be first used to undertake analysis of real-size solar cells within the framework of the ESA's JUICE mission.

\section{THE LARGE SURFACE IRRADIATED-CELL}

\subsection{Setup description}

The LSIC device allows irradiation of a wide variety of samples up to a A5 size paper dimension. It includes the possibility to perform in situ 4-wire electrical measurements, (1) in the dark, or under illumination through a window made of radiation-resistant quartz glass (2) (Fig. 1). The setup can be coupled to the SIRIUS beamline by using a metal piece (3) including a water-cooled removable stopper actionable with a hydraulic piston to stop incident electrons.
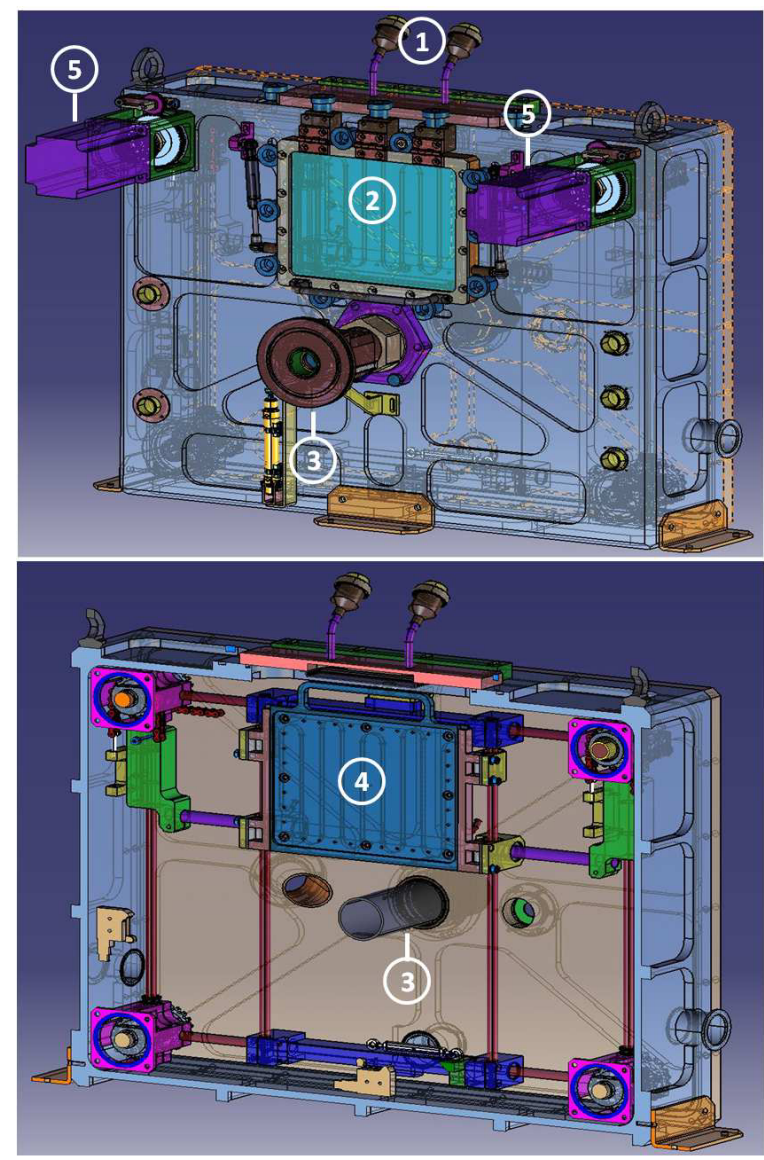

Figure 1. 3D views of the large surface cell device. (a) Back-side and (b) front-side without the hermetic lid.

The principle of the experiment consists of scanning the sample holder (4) on the electron beamline. Two servomotors (5) control the movement of pulleys and chains allowing a fine and smooth XY displacement of the sample holder.

A LabVIEW program drives the motion of the sample holder and manages a complete irradiation cycle. The surface to be irradiated, with the appropriate parameters (electron beam energy, fluence, current and temperature of irradiation) can be set easily and precisely. The displacement velocity of the sample holder is enslaved to a digital charge integrator to ensure a good homogeneity in terms of electron fluence all over the irradiated area.

The sample holder, shown in Fig. 2, is equipped with an adjustable system (6) intended to ensure both the positioning and maintaining in place of several samples simultaneously (7). This assembly made of conductive and insulating pieces includes grooves to guide wires in order to perform up to 4 4-wire in situ electrical measurements in a same configuration of experiments.

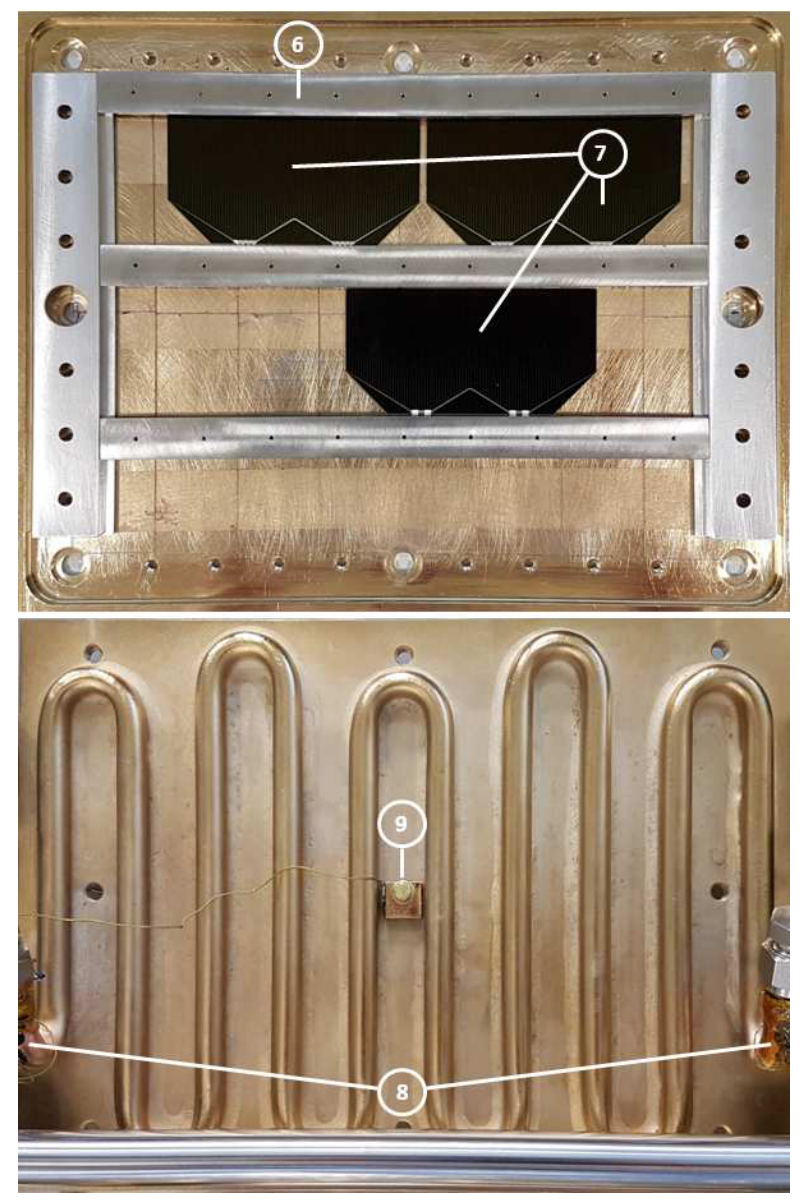

Figure 2. Pictures of the sample holder: (a) front-side and (b) back-side with the welded liquid nitrogen heat exchanger.

Liquid nitrogen circulating in a coil welded on the opposite side of the sample holder allows to work in a wide range of temperatures ranging from $100 \mathrm{~K}$ to $300 \mathrm{~K}$ (Fig. 2). A specific automatic proportional-integralderivative algorithm is used to regulate the temperature by means of a coaxial thermocouple located the entrance of the heat exchanger. Temperatures values at the entrance and exit of the coil are obtained with 2 Cernox sensors (8). A third one is screwed at the middle of the 
sample holder (9) to get the temperature of samples during experiments.

\subsection{Project Schedule Development}

The qualification phase of the LSIC device has started in July 2016 and some results are discussed in the next part of this paper. Irradiation experiments with in situ electrical measurements will be performed by the end of this year. The public availability of the setup is planned for early 2017.

\section{PRELIMINARY RESULTS}

\subsection{Sample holder displacement}

Due to the harsh radiation environment during experiments at the SIRIUS facility, electronic components of the LSIC setup have to be deported a few meters from the beamline. The use of two-conductor shielded twisted pair cables reduces drastically electromagnetic interferences that is usually a major source of problems for systems using servomotors. Online tests have shown that the sample holder can be driven instantly and precisely with the LabVIEW control program (Fig. 3). This allows to choose the motion speed and velocity, as well as the scanning frequency of the sample holder.

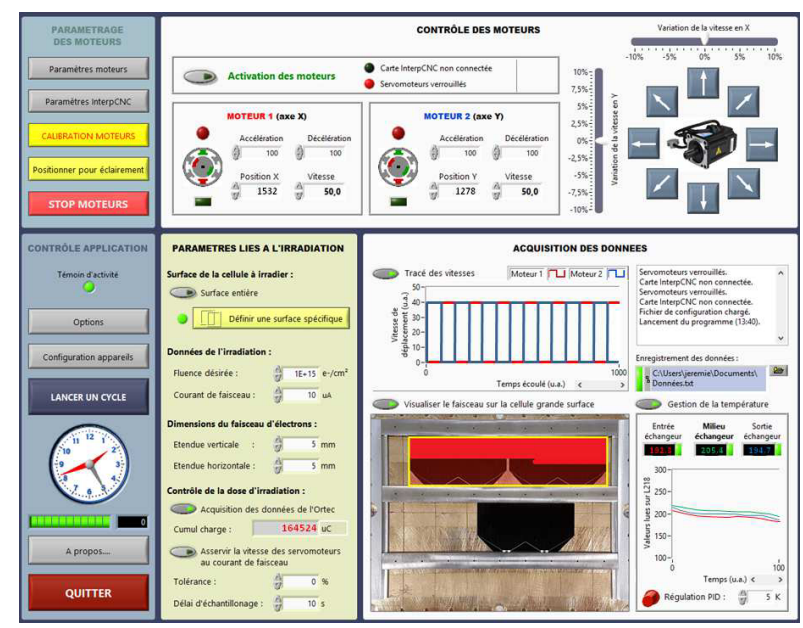

Figure 3. LabVIEW control program of the LSIC device.

The relative position of the sample holder on the electron beamline during irradiation experiments is represented in the LabVIEW interface by a red spot (Fig. 3). It includes an alpha component that allows to visualize the displacement history of the sample holder. This ensure an homogeneous irradiation all over the surface to be irradiated (Fig. 3).

\subsection{Temperature control}

Preliminary tests performed with the heat exchanger have shown a very satisfactory behavior in terms of cooling power capability of the LSIC device, since the sample holder can be cooled from RT to $80 \mathrm{~K}$ in around $30 \mathrm{mn}$ (Fig.3).

Cooling curves of the sample holder
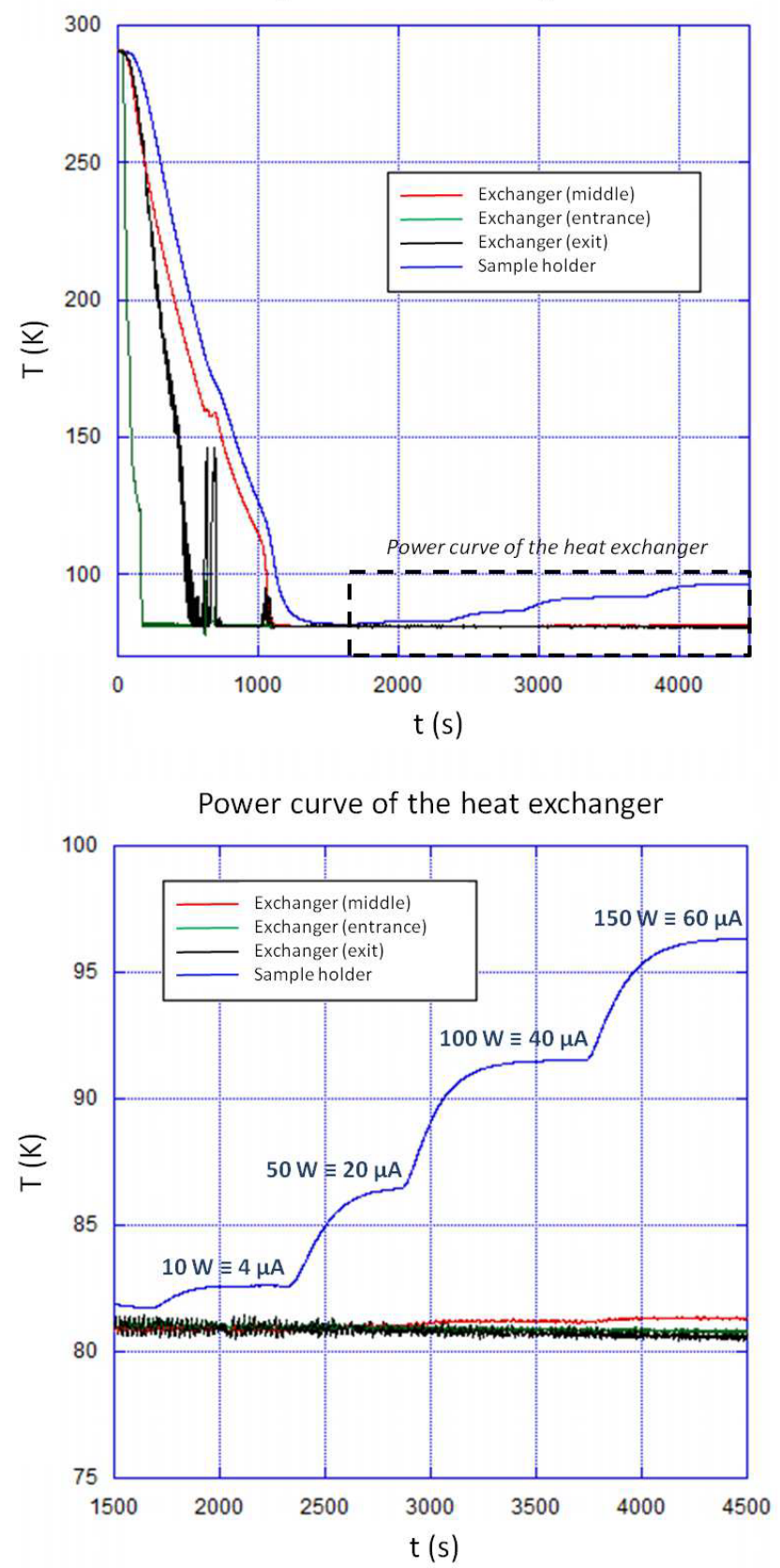

Figure 4. (up) Cooling down curves of the sample holder and (down) power curve of the heat exchanger.

High flux irradiation with a high beam current is expected to cause significant increase in sample temperature because of vast beam heating. This effect is due to the lost 
since electrons lose their energy mostly by electronic intraction in materials. The heating power transferred to a sample during irradiation $\left(P_{t h}\right)$ can be written as follows:

$$
P_{t h}=P_{e l}-P_{R}
$$

with $P_{e l}$ as the power of the incident electrons, and $P_{R}$ as the power lost through $\mathrm{X}$-ray radiation.

In order to simulate the power of the electron beam, a heating resistance was screwed of the sample holder. The $P_{e l}$ parameter can be expressed as follows:

$$
P_{e l}=U . I
$$

with $U$ as the energy of incident electrons in $\mathrm{MeV}$, and $I$ as the current beam in $\mu \mathrm{A}$. In a conservative approach, $P_{R}$ is not taken into account, so that the current beam can be directly deduced from the power supplied to the resistance.

Fig. 5 shows that the sample holder temperature is below $100 \mathrm{~K}$ for current beam up to $60 \mu \mathrm{A}$ that is a significantly high value.

\section{REFERENCES}

[1] http://emir.in2p3.fr/-Le-reseau-EMIR- 